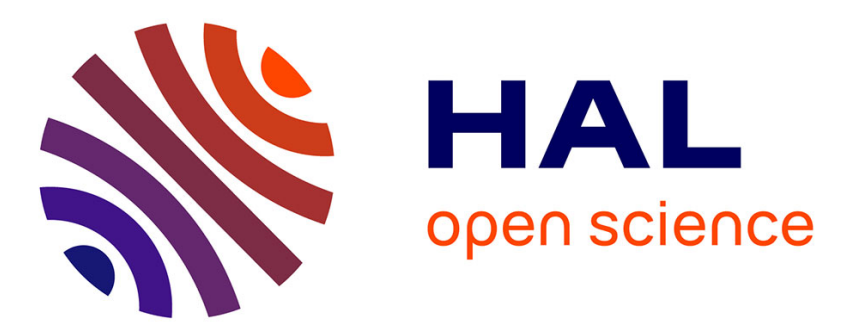

\title{
Tuning of the Optical Properties of the Transparent Conducting Oxide SrVO3 by Electronic Correlations
}

Alexis Boileau, Aïmane Cheikh, Arnaud Fouchet, Adrian David, Christophe Labbé, Philippe Marie, Fabrice Gourbilleau, Ulrike Luders

\section{- To cite this version:}

Alexis Boileau, Aïmane Cheikh, Arnaud Fouchet, Adrian David, Christophe Labbé, et al.. Tuning of the Optical Properties of the Transparent Conducting Oxide SrVO3 by Electronic Correlations. Advanced Optical Materials, 2019, 7 (7), pp.1801516. 10.1002/adom.201801516 . hal-01998780

\section{HAL Id: hal-01998780 \\ https://hal.science/hal-01998780}

Submitted on 29 Jan 2019

HAL is a multi-disciplinary open access archive for the deposit and dissemination of scientific research documents, whether they are published or not. The documents may come from teaching and research institutions in France or abroad, or from public or private research centers.
L'archive ouverte pluridisciplinaire HAL, est destinée au dépôt et à la diffusion de documents scientifiques de niveau recherche, publiés ou non, émanant des établissements d'enseignement et de recherche français ou étrangers, des laboratoires publics ou privés. 


\title{
Tuning of the Optical Properties of the Transparent Conducting Oxide $\mathrm{SrVO}_{3}$ by Electronic Correlations
}

\author{
Alexis Boileau, Aïmane Cheikh, Arnaud Fouchet, Adrian David, Christophe Labbé, \\ Philippe Marie, Fabrice Gourbilleau, and Ulrike Lüders*
}

The vanadate $\mathrm{SrVO}_{3}$ is a transparent conductor perovskite with optical and electrical properties competing with those of the most-used indium tin oxide material. Although its charge density is comparable to that of metals, $\mathrm{SrVO}_{3}$ shows a plasma frequency below the visible range due to strong electronic correlations characterizing the electronic transport in this material and enhancing the effective mass. Therefore, the well-known interplay between the structure and the electronic properties of strongly correlated systems can be used in such transparent conductor to tune the optical properties, as the plasma frequency also depends on the effective mass. In this study, $\mathrm{SrVO}_{3}$ films are grown by pulsed laser deposition onto different lattice mismatched perovskite substrates such as $\mathrm{SrTiO}_{3}, \mathrm{LaAlO}_{3}$, and $\left(\mathrm{LaAlO}_{3}\right)_{0.3}\left(\mathrm{Sr}_{2} \mathrm{TaAlO}_{6}\right)_{0.7}$ at different growth temperatures. The structural, electronic, and optical properties are analyzed, illustrating the influence of the strain on the structure of the films and on a shift of the plasma frequency. The electronic correlations in this new group of transparent conducting oxides can be therefore used as a supplementary lever for the tuning of the functional properties.

\section{Introduction}

$\mathrm{SrVO}_{3}$ (SVO) is one of the strongly correlated metals that were recently identified to be transparent conducting oxides (TCO). ${ }^{[1]}$ The demonstrated properties of this system stand up to those of the standard indium tin oxide material, providing therefore a valuable alternative for a number of applications. Although it was shown that the amorphous phase of SVO is not conducting, ${ }^{[2]}$ the potential of application stays important in adapted systems due to the absence of indium and its price fluctuations related to the limited availability. An intriguing aspect

Dr. A. Boileau, A. Cheikh, Dr. A. Fouchet, Dr. A. David, Dr. U. Lüders CRISMAT (Laboratoire de Cristallographie et Science des Matériaux) CNRS UMR6508 - ENSICAEN

Normandie Université

14050 Caen Cedex 4, France

E-mail: ulrike.luders@ensicaen.fr

Dr. C. Labbé, Dr. P. Marie, Dr. F. Gourbilleau

CIMAP (Centre de Recherche sur les lons, les Matériaux et la

Photonique)

ENSICAEN - UNICAEN - CNRS

Normandie Université

14050 Caen Cedex 4, France

The ORCID identification number(s) for the author(s) of this article can be found under https://doi.org/10.1002/adom.201801516. of SVO as a TCO is the fact that although the charge density is that of typical metals (in the range of $10^{22} \mathrm{~cm}^{-3}$ ), the screened plasma frequency $\omega_{\mathrm{p}}$ is pushed out of the visible range due to the strong electronic correlations enhancing the effective mass $m *$. Consequently, the electronic correlations do not only govern the electronic transport properties, but also the optical ones in the visible range.

Such interplay between the electronic and optical properties via the electronic correlations may also open a new way for the optimization of the functional properties of SVO. In strongly correlated metals, it is known that strain may influence the correlation characteristics via the related crystal distortions, ${ }^{[3-5]}$ which were also predicted theoretically for SVO.[6,7] In a strongly correlated TCO as SVO, it may therefore be possible to not only influence the electrical transport by strain, but also the optical properties. Although the structural and electronic properties of SVO were studied intensely in the thin film ${ }^{[8-12]}$ or in superlattices form, ${ }^{[10,13]}$ no comparative study of SVO films on different substrates inducing variation of strain was published, neither its influence on the optical properties. The aim of this study is therefore to use three substrates, on which epitaxial growth of SVO is possible, in order to elucidate the influence of the strain on both the electronic and the optical properties.

However, in the discussion of the relationship between structure and properties in transition metal oxide thin films, the stoichiometry of the films has also to be taken into account. Especially in SVO, it has been shown that both a cationic ${ }^{[9]}$ as also an anionic nonstoichiometry ${ }^{[11,12,14,15]}$ play an important role in the properties. The management of the oxygen content is a special issue in SVO due to the existence of some oxides with higher oxidation state of vanadium such as $\mathrm{Sr}_{3} \mathrm{~V}_{2} \mathrm{O}_{8}$ or $\mathrm{Sr}_{2} \mathrm{~V}_{2} \mathrm{O}_{7}$, being thermodynamically more stable than the perovskite structure. ${ }^{[16]}$ This implies that widely used mechanisms to influence on the oxygen stoichiometry of thin films, as the growth in different oxygen pressures or postannealing treatments, are not possible or have to be controlled closely in order to avoid the formation of the orthovanadate, which is transparent but insulating. Even more, recently it has been shown in a closely related system, $\mathrm{SrCoO}_{3}$, that strain and oxygen vacancies show a complex interplay: ${ }^{[17]}$ in films undergoing a tensile strain, the density of oxygen vacancies is enhanced, 
probably due to the weakening of the $\mathrm{Co}-\mathrm{O}$ bonds. This observation seems to be part of more general tendency in perovskite oxides, where the introduction of oxygen vacancies is favored by tensile strain allowing for an accommodation of the oxygen vacancy volume. ${ }^{[18]}$ In order to estimate the effects of oxygen vacancies, which cannot be measured with easy means in thin films, we have included also a study on the growth temperature $T_{G}$. The films, being deposited in vacuum to avoid in situ overoxidation, undergo a strongly reducing atmosphere at least at high $T_{G}$, while at low $T_{G}$ the oxygen transport in the film is less activated. By comparing the films at different $T_{\mathrm{G}}$, it will be possible to elucidate the role of oxygen vacancies on the functional properties of the film, although it will not be possible to gain absolute knowledge.

In this work, we report on the experimental investigation of the growth of $\mathrm{SrVO}_{3}$ films on different perovskite substrates and their influence on the structural, electrical, and optical properties. The selected substrates are (001) oriented $\mathrm{SrTiO}_{3}$ (STO), $\left(\mathrm{LaAlO}_{3}\right)_{0.3}\left(\mathrm{Sr}_{2} \mathrm{TaAlO}_{6}\right)_{0.7} \quad(\mathrm{LSAT})$, and $\mathrm{LaAlO}_{3}$ (LAO) monocrystals, adapted to the growth of SVO. The lattice parameter of STO, LSAT, and LAO substrates is 3.905, 3.868, and $3.790 \AA$, which lead to a lattice mismatch with SVO bulk (3.840 A) of $1.69 \%, 0.73 \%$, and $-1.30 \%$, respectively. Therefore, SVO films grown on STO and LSAT are tensile-strained while the films grown on LAO are compressive-strained. The aim of this study is to determine the functional properties for films undergoing the different strain states and understand the role of these strains.

\section{Results and Discussion}

\subsection{Structural Characterizations}

First, the picture of deposited films on STO, LAO, and LSAT displayed in Figure 1a gives an overview of the good transparency of SVO films for a thickness of $30 \mathrm{~nm}$. Concerning X-ray diffraction (XRD) measurements, Figure 1b shows specular $\theta-2 \theta$ diffractograms around the (002) Bragg peak of SVO films grown on STO and LAO between 300 and $700{ }^{\circ} \mathrm{C}$ (the data concerning the films on LSAT were published elsewhere $\left.{ }^{[2]}\right)$. At $300{ }^{\circ} \mathrm{C}$, only the peak attributed to the $(002)$ reflection arising from the substrate is visible at $46.47^{\circ}$ and $47.97^{\circ}$ for STO and LAO, respectively. The peak due to the (002) reflection of the SVO cubic structure appears around $47.25^{\circ}$, and $46.50^{\circ}$ on STO and LAO, respectively, between 300 and $400{ }^{\circ} \mathrm{C}$. Similar results were found for the films on LSAT. This crystallization temperature of SVO is remarkably low and does not seem to depend on the substrate, at least in the defined temperature interval. From the observed Laue fringes, the layer thickness was determined to be in the range of $37-40 \mathrm{~nm}$ by simulations (not shown). As the presence of Laue fringes can be considered as a marker of the quality of the crystalline local order, highly crystallized films are grown at $400{ }^{\circ} \mathrm{C}$ on STO and LSAT with a flat film/substrate interface. By contrast, the film deposited on LAO at the same temperature does not exhibit Laue fringes. In the case of LSAT, the lattice mismatch is limited to $0.72 \%$ but increases positively up to $1.66 \%$ for STO and negatively down to $-1.32 \%$ for LAO. Given that the epitaxial
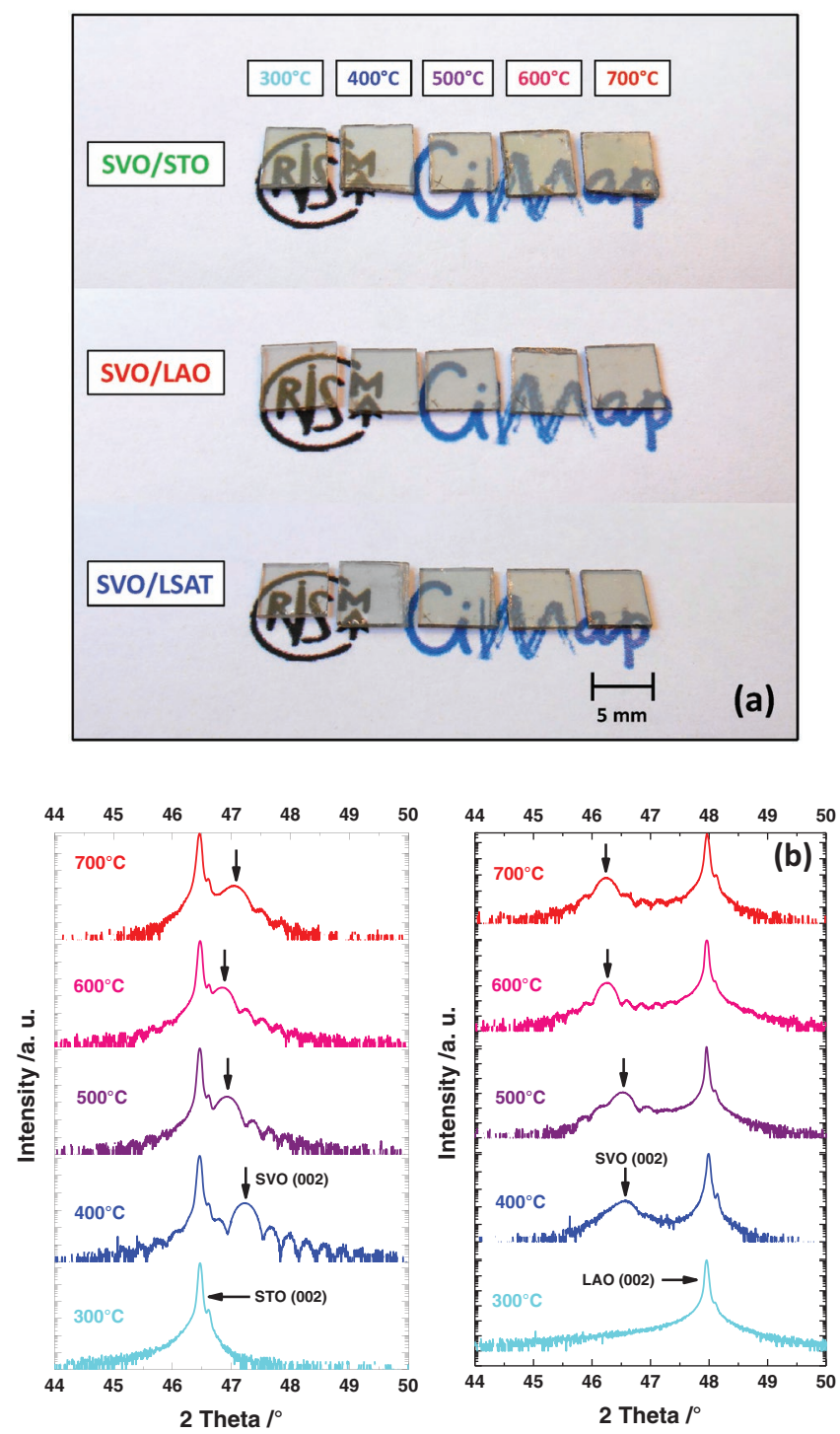

Figure 1. a) Image of SVO films grown onto STO, LAO, and LSAT between 300 and $700^{\circ} \mathrm{C}$. b) $\theta-2 \theta \mathrm{X}$-ray diffractograms in the vicinity of the (002) reflection of SVO thin films on STO and LAO grown between 300 and $700{ }^{\circ} \mathrm{C}$. For SVO/LSAT samples refer to ref. [2].

tensile strain induced by the STO substrate is more important than the compressive one induced by LAO, the loss of oscillations may indicate the negative impact of compressive strains on the crystallization of SVO. The energy required for the epitaxial stabilization of SVO in compressive stress is therefore partially reinvested in the elastic potential energy required to induce the deformation of the perovskite structure by the apical elongation of the octahedra of the corner-sharing perovskite structure.

As $T_{G}$ is increased, the amplitude of the Laue fringes around the (002) SVO peak deposited on LAO significantly increases as the provided thermal energy becomes high enough to ensure both the deformation and the crystallization of the structure. Consequently, the quality of the crystallization of SVO at low temperature can be strongly modulated by the deformation of the strained epitaxial structure imposed by 

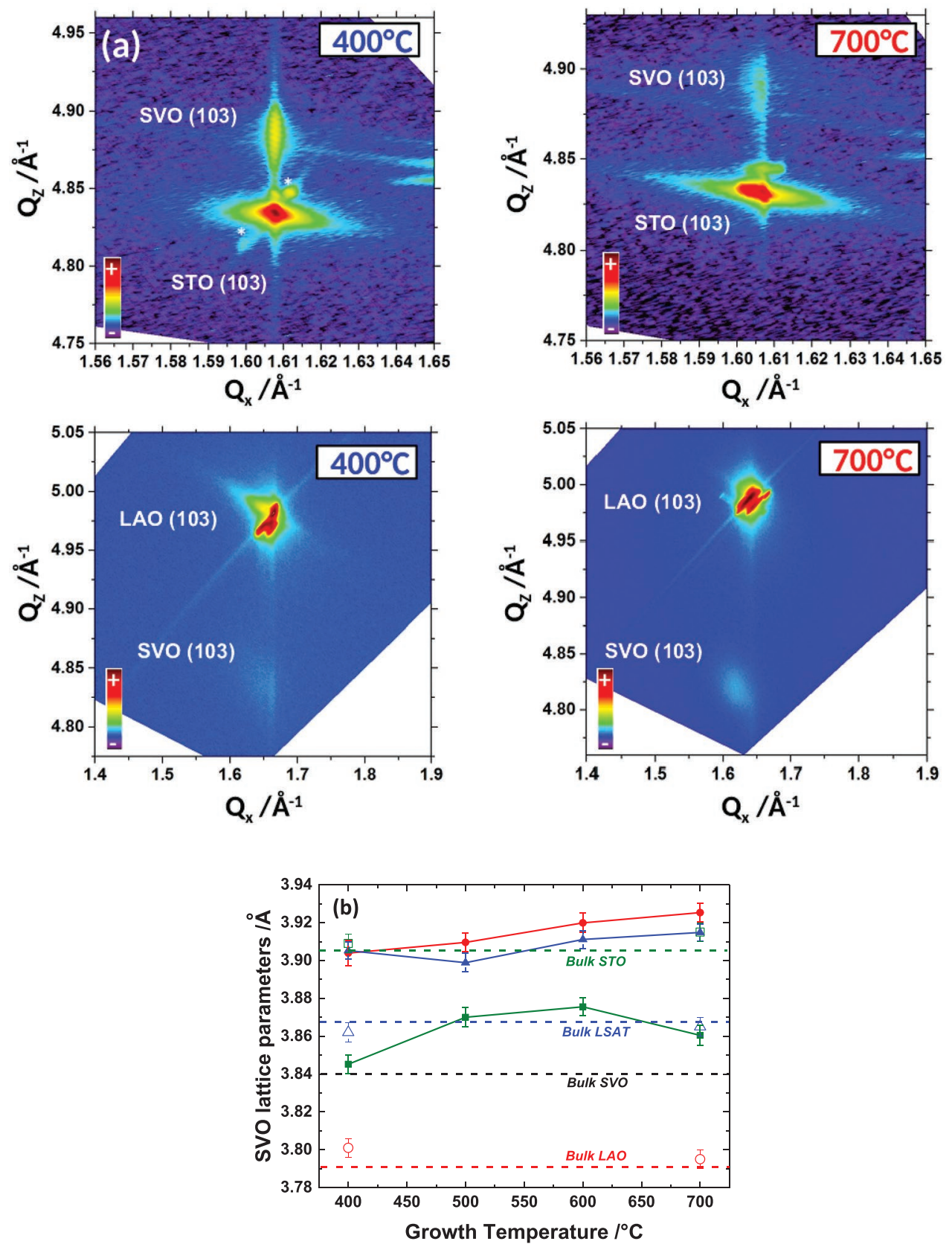

Figure 2. a) Reciprocal space maps around the (103) cubic reflection of SVO thin films grown at 400 and $700{ }^{\circ} \mathrm{C}$ on STO and LAO substrates. The reflections marked by a star $\left(400^{\circ} \mathrm{C}\right)$ are artefacts due to the diffractometer setup. b) Out-of-plane (solid symbols) and in-plane (open symbols) lattice parameters evolution as a function of the growth temperature of SVO films on STO (red squares), LSAT (blue triangles), and LAO (red circles).

the substrate and widely enhanced by implication of a biaxial tensile strain.

To get better insight in the in-plane structural relation between SVO and the substrates, asymmetric film reflections were also measured in skew symmetry. In Figure 2a, reciprocal space maps (RSMs) around the (103) Bragg peaks for the film on STO are presented for $T_{G}$ at 400 and $700{ }^{\circ} \mathrm{C}$, representative also for the other used substrates. From the vertical alignment of the film and substrate peaks at the same $Q_{x}$ value, we can note that SVO films are fully strained to the substrate regardless of growth temperature for STO and slightly relaxed for LAO.
From the (002) and (103) peaks, we have determined the evolution of in-plane and out-of-plane cubic cell parameters $a_{\mathrm{f}}$ and $c_{\mathrm{f}}$ of SVO films depending on the growth temperature. The corresponding lattice parameters are displayed in Figure $2 \mathrm{~b}$. The in-plane lattice parameter $a_{\mathrm{f}}$ (open symbols) of SVO is found to be 3.90(9)-3.91(5), 3.86(2)-3.86(5), and 3.80(1)-3.79(5) $\AA$ at 400 and $700{ }^{\circ} \mathrm{C}$ on STO, LSAT, and LAO, respectively, whereas one of the substrates $a_{\mathrm{s}}$ is equal to $3.90(5), 3.86(8)$, and $3.79(0)$ $\AA$ for bulk substrates. Therefore, as observed earlier in the RSMs, $a_{\mathrm{f}}$ matches closely the substrate lattice parameter for all films, independently of the induced strain. 
However, the out-of-plane lattice parameter $c_{f}$ (solid symbols) exhibits a different evolution with $T_{G}$ and depends strongly of the nature of the substrate. For SVO//STO, $c_{\mathrm{f}}$ increases rapidly from $3.84(5)$ to $3.87(5) \AA$ as the $T_{G}$ increases up to $600{ }^{\circ} \mathrm{C}$. It can be noticed that for the film deposited at $400{ }^{\circ} \mathrm{C}$, the $c$-axis is close to the value reported for bulk SVO, found to be between 3.82 and $3.84 \AA .{ }^{[19-22]}$ Around $600{ }^{\circ} \mathrm{C}, c_{f}$ goes through a maximum and decreases slightly down to $3.86 \AA$ at $700{ }^{\circ} \mathrm{C}$. The expansion of the $c$-axis observed between 400 and $600{ }^{\circ} \mathrm{C}$ may be related to the creation of oxygen vacancies in SVO due to the growth under vacuum. The incorporation of oxygen vacancies induces a swelling effect of the cell driven by the reduction of $\mathrm{V}^{4+}$ to the larger cation $\mathrm{V}^{3+}$ : the ionic radius increases slightly from 0.58 to $0.64 \AA .{ }^{[23]}$ Actually, the near-stagnation of the $c$-axis of SVO//STO at high $T_{\mathrm{G}}$ (whereas it increases continuously for LAO and LSAT) can be understood by the vicinity of the STO substrate. Heating of the STO under vacuum is known to generate oxygen vacancies in the structure and to create colorcenters. ${ }^{[24-26]}$ Conversely, SVO is highly sensitive to oxygen capture even for reduced oxygen partial pressures of $10^{-4} \mathrm{~Pa}^{[12]}$ At $T_{\mathrm{G}}$ above $500-600^{\circ} \mathrm{C}$, the thermal energy is high enough to activate the oxygen diffusion at the interface from STO toward SVO and compensate the oxygen depletion in the film created by the reducing growth conditions. Consequently, the substrate can be viewed as an oxygen reservoir for the film during the growth. Thus, a change of $T_{\mathrm{G}}$ would lead to a modification of the vacancy doping in STO and SVO, the film being a source of oxygen vacancies, as proposed also by Herranz et al. ${ }^{[27]}$

For SVO//LAO films, $c_{\mathrm{f}}$ is remarkably shifted toward high values to accommodate compressive strains induced by the LAO misfit. However, LAO is stable under vacuum conditions and the reduction of the substrate does not occur as much as in STO one. In these conditions, we observe that $c_{\mathrm{f}}$ rises continuously from 3.904 to $3.925 \AA$ when the deposition temperature is increased, indicating the creation of oxygen vacancies in SVO for higher $T_{\mathrm{G}}$. At the same time, the crystallinity of the films is improved due to the increased thermal energy.

For SVO//LSAT, we observe a similar evolution of $c_{\mathrm{f}}$ with $T_{\mathrm{G}}$. The $c$-axis lattice parameter increases from 3.905 to $3.915 \AA$ as observed on the other substrates, but the high observed values, similar to SVO deposited on LAO, is somewhat unexpected due to the change of the misfit from compressive on LAO to slightly tensile on LSAT. If the reaction of the film on the strain would be purely elastic, values smaller than the bulk would be expected on LSAT. It is known that a deviation in the cation stoichiometry of the perovskite could influence the lattice parameters of films. Studies of SVO thin film on LSAT substrate have also variations between 3.82 and $3.84 \AA$ depending on the $\mathrm{Sr}$ and $\mathrm{V}$ content. ${ }^{[9]}$ The reported values are much smaller than those observed in this study, and Rutherford backscattering spectrometry (RBS) showed a $\mathrm{Sr} / \mathrm{V}$ ratio of about 1 , so that this effect can be ruled out here. However, it was shown that the presence of biaxial tensile stain may induce an oxygen depletion to satisfy the deformation of the octahedral network. Recently, Petrie et al. ${ }^{[17]}$ have shown a similar behavior in $\mathrm{SrCoO}_{3}$ films, where the creation of oxygen vacancies was shown to be facilitated by the introduction of tensile strain, probably due to weakened $\mathrm{M}-\mathrm{O}$ bonding.
Therefore, coherently strained films were observed on all substrates, permitting to study the influence of the strain on the physical properties. However, care has to be taken with the films on STO, where an important influence from possible oxygen vacancies cannot be excluded.

\subsection{Transport Properties}

\subsection{Transport Properties of As-Grown Films}

The temperature dependence of the electrical resistivity of the films was measured between 5 and $300 \mathrm{~K}$. Results are reported in Figure 3 for STO, LAO and LSAT at different $T_{\mathrm{G}}$. For all crystallized films, we observe a metallic behavior in the whole temperature range in good agreement with earlier works. ${ }^{[1,15]}$ Globally, the resistivity measured at room temperature is between 1.10 and $2.37 \times 10^{-4} \Omega \mathrm{cm}$ and corresponds to the typical order of magnitude observed for SVO films deposited by pulsed laser deposition (PLD). ${ }^{[28,29]}$ Despite large differences observed in the structural quality of films depending on the growth temperature and the nature of the substrate, the resistivity remains in the same order of magnitude and does not vary much at room temperature. At most, we observe a varia-

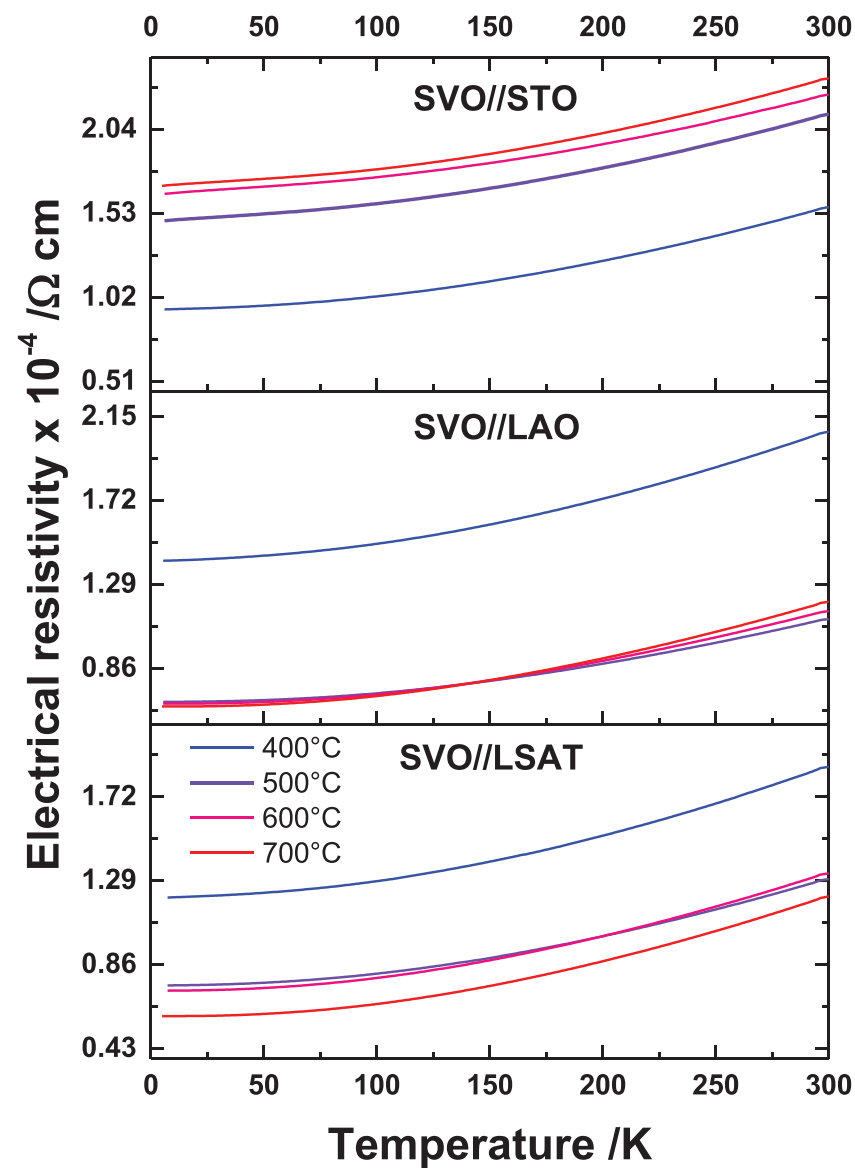

Figure 3. Temperature dependence of the electrical resistivity of SVO films grown on STO, LAO, and LSAT substrates between 400 and $700{ }^{\circ} \mathrm{C}$. Data in the bottom panel are reproduced from Boileau et al.[2] 

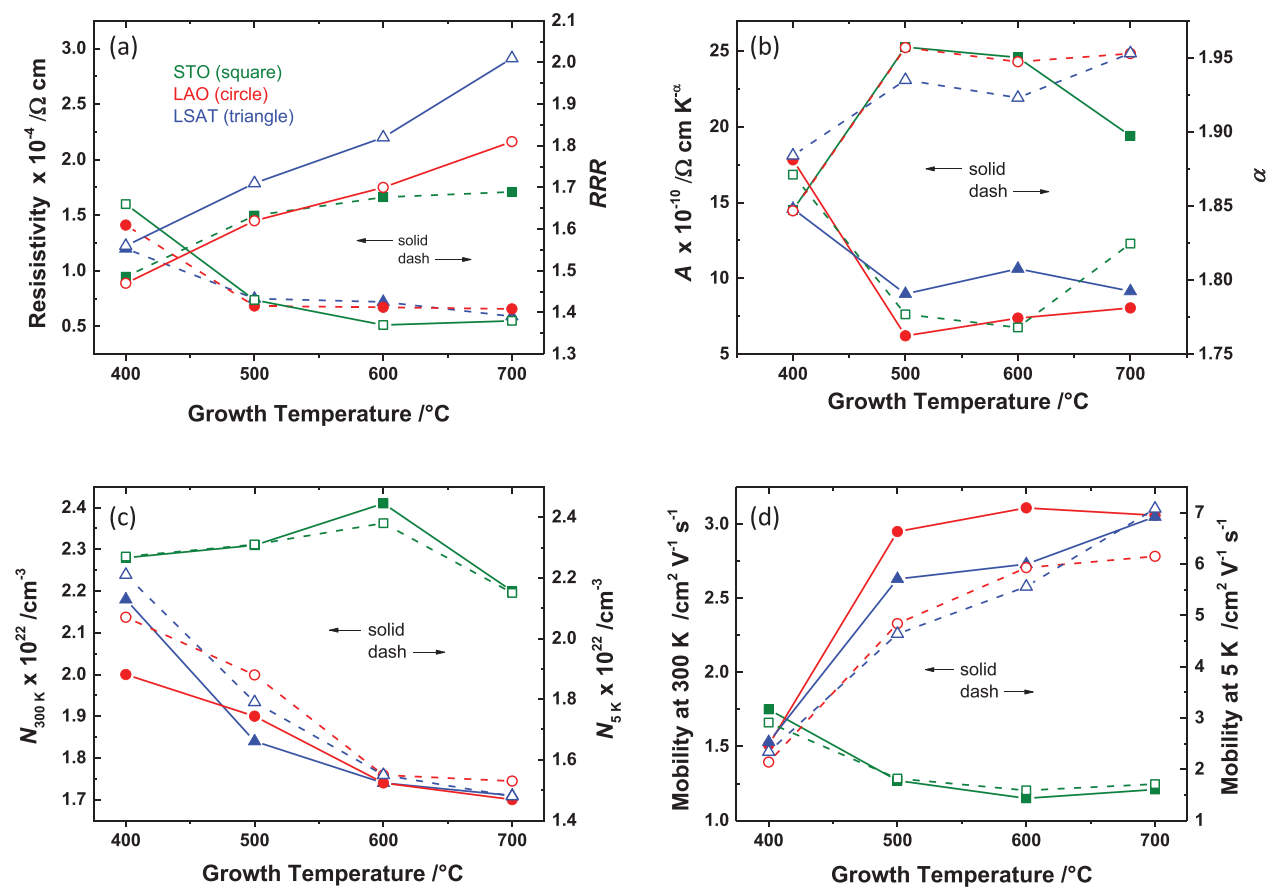

Figure 4. Temperature dependence of transport properties of SVO film grown on STO (green squares), LAO (red circles), and LSAT (blue triangles). a) Electrical resistivity at $5 \mathrm{~K}$ and the RRR ratio, b) $A$ and $\alpha$ coefficients, c) the density of free carriers at 300 and $5 \mathrm{~K}$, and d) the mobility of free carriers at 300 and $5 \mathrm{~K}$.

tion in the resistivity of a factor of two. Hence, the influences of the substrate and $T_{\mathrm{G}}$ are relatively small compared to the need of well-defined crystalline perovskite structure for good transport properties, as was also pointed out for thin films on LSAT. $^{[2]}$

However, regarding the details of the transport behavior, differences due to the substrates can be observed. In Figure 4, different markers for all substrates and the different growth temperatures are combined. In Figure 4a, the electrical resistivity measured $5 \mathrm{~K}\left(\rho_{5 \mathrm{~K}}\right)$ as well as the residual resistivity ratio (RRR) defined as the ratio between $\rho_{300 \mathrm{~K}}$ and $\rho_{5 \mathrm{~K}}$ are displayed. The temperature dependence of the resistivity was fitted on the basis of the typical model for metallic conductors $\rho(T)=\rho_{0}+$ $A \times T^{\alpha}$ where $\rho_{0}$ is the residual resistivity taken at $0 \mathrm{~K}$ and $A$ is related to the electronic interactions for a strongly correlated metal. The exponent $\alpha$ gives an indication on the Fermi liquid character of the system. ${ }^{[10,15,30]}$ The fit results are shown in Figure $4 \mathrm{~b}$. To complete electrical transport data, the density and the mobility of carriers measured by Hall effect at 300 and $5 \mathrm{~K}$ are reported in Figure $4 c, d$, respectively.

For the films on LAO and LSAT, the evolution of the different markers with $T_{\mathrm{G}}$ is remarkably similar. The resistivity decreases in both cases with the increase of $T_{\mathrm{G}}$, leading to an increase of the RRR. For high $T_{\mathrm{G}}$, the fit parameters of the temperature dependence of the resistivity are near to what was observed in the bulk material, ${ }^{[31]} \alpha=2$ and $A=5.9 \times 10^{-10} \Omega \mathrm{cm} \mathrm{K}^{-2}$. Regarding the characteristics of the charge carriers, the charge density $N$ decreases with $T_{\mathrm{G}}$, reaching a value about between $1.5(5 \mathrm{~K})$ and $1.7 \times 10^{22} \mathrm{~cm}^{-3}(300 \mathrm{~K})$ for the samples grown at $700{ }^{\circ} \mathrm{C}$. This value is consistent with the expected value based on the hypothesis that each $\mathrm{V}^{4+}$ ion $\left(3 \mathrm{~d}^{1}\right)$ contributes one electron, which would add up to $N=1.77 \times 10^{22} \mathrm{~cm}^{-3}$ with the bulk density. The mobility $\mu$ increases with $T_{\mathrm{G}}$, reaching values of $6-7 \mathrm{~cm}^{2} \mathrm{~V}^{-1} \mathrm{~s}^{-1}$ at $5 \mathrm{~K}$ for $T_{\mathrm{G}}=700{ }^{\circ} \mathrm{C}$. These values are in the same range as those observed in other studies of thin films on LSAT substrates, outreaching those of other films grown by $\mathrm{PLD},{ }^{[10]}$ but staying about a factor of two (at room temperature) lower than those of molecular beam epitaxy (MBE) grown samples. ${ }^{[1,9]}$

The films on LAO and LSAT show therefore charge transport characteristics, which are comparable to bulk properties at high $T_{\mathrm{G}}$, while for low $T_{\mathrm{G}}$, the markers related to disorder $\left(\rho_{5 \mathrm{~K}}, \mathrm{RRR}\right.$, $\alpha$, and $\mu$ ) show stronger deviations. Thus, the observed evolution of the resistivity with $T_{\mathrm{G}}$ can be explained by the decrease of disorder and therefore to the increase in crystalline quality, as was observed in the XRD study on LAO. However, for the films grown on LSAT, an exceptionally high crystalline quality is already observed at $400{ }^{\circ} \mathrm{C},{ }^{[2]}$ comparable to the one of the film grown at $700{ }^{\circ} \mathrm{C}$, which does not seem consistent with structural disorder being at the origin of the degradation of the electronic transport. Moyer et al. ${ }^{[9]}$ have shown that an imbalance of the Sr-V stoichiometry may also be at the origin of high residual resistivities and low mobilities, but the RBS analysis of the films of this study did not show an evolution for different $T_{\mathrm{G}}$ or different substrates. The origin of the observed scattering may therefore also be related to non-crystalline disorder, i.e., purely electronic disorder, which will be addressed in future studies of the system.

The films grown on STO show a different behavior compared to the films grown on LAO and LSAT. While for the sample grown at $400{ }^{\circ} \mathrm{C}$, the resistivity is still comparable to the films grown in LAO and LSAT, $\rho$ increases for higher $T_{\mathrm{G}}$ and the RRR decreases. This evolution is also observed for $A$ and $\alpha$, where the characteristics are comparable to the films on 
LAO and LSAT at $400{ }^{\circ} \mathrm{C}$, but while these latter approach the bulk properties at higher $T_{G}$, the films on STO deviate more strongly from the bulk values, with $\alpha$ decreasing down to 1.77 at $T_{\mathrm{G}}=600{ }^{\circ} \mathrm{C}$. $A$ increases in the same temperature range, resulting in a value about four times larger than the bulk value. Interestingly, the film grown at $700{ }^{\circ} \mathrm{C}$ reverses the tendencies for the low $T_{\mathrm{G}}$ samples, probably due to the starting exchange of the oxygen vacancies between the substrate and the film, as was observed in the structural properties. $N$ stays roughly constant in the observed $T_{\mathrm{G}}$ range, and $\mu$ decreases, indicating that it is not a lack of charge carriers, but rather a lack of mobility leading to the high resistivity at high $T_{\mathrm{G}}$.

It seems therefore that the strain plays only an indirect role for the electronic properties, as the films on LAO (large compressive strain) and LSAT (small tensile strain) show similar behaviors, while the properties of the films on STO (large tensile strain) are governed rather by the oxygen stoichiometry in the film. This interpretation is underlined by the fact that the samples grown at $400{ }^{\circ} \mathrm{C}$ show similar properties on all substrates, at a growth temperature where the thermodynamic reduction of the film during the growth should be rather small. If the strain would have a direct influence, these films, fully strained, should show pronounced differences. On the other hand, the strain seems to have an indirect influence via the oxygen vacancies: for increasing $T_{\mathrm{G}}$, the probability of the introduction of oxygen vacancies in the films becomes more important for all films, but the ones grown on LAO and LSAT show actually a tendency to more bulk-like properties, excluding therefore a high oxygen vacancy density. For STO, the introduction of oxygen vacancies is favored both by the high tensile strain and by $T_{\mathrm{G}}$ up to $600{ }^{\circ} \mathrm{C}$, while at higher temperature the oxygen exchange between the substrate and the film sets in. The transport characteristics reflect this evolution, illustrating the importance of the oxygen vacancies and therefore indirectly of the tensile strain for the films on STO.

Turning back to the initial motivation of the study, the influence of the substrate on the electronic correlations can be estimated using the fit parameter $A$ and the charge density $N$. In a simplified model, ${ }^{[31]} A^{1 / 2} N^{-1 / 3}$ is proportional to the effective electronic mass $m^{*}$, one of the governing parameters also for the optical properties. In Figure 5, the values of the coefficient $A^{1 / 2} N^{-1 / 3}$ for the different substrates and $T_{\mathrm{G}}$ are shown, confirming the observed differences in the transport properties of the films grown on STO and those grown in LAO and LSAT. While at $T_{\mathrm{G}}=400{ }^{\circ} \mathrm{C}$, the values are similar for all substrates, $A^{1 / 2} N^{-1 / 3}$ enhances for films grown on STO for higher $T_{\mathrm{G}}$, while they decrease for films grown on LAO and LSAT. The strongest difference is observed for the samples grown at $T_{\mathrm{G}}=500{ }^{\circ} \mathrm{C}$, with a ratio in the order of 2 , indicating a value of $m *$ twice as high on STO compared to the other substrates. The observed structural changes have therefore an influence on the electronic correlations of the SVO films.

\subsubsection{Optical Properties of As-Grown Films}

Optical transmission spectra were acquired in the visible range. The transmittance taken in the middle of the visible window at $500 \mathrm{~nm}$ is shown in Figure 6a for the films grown on STO and

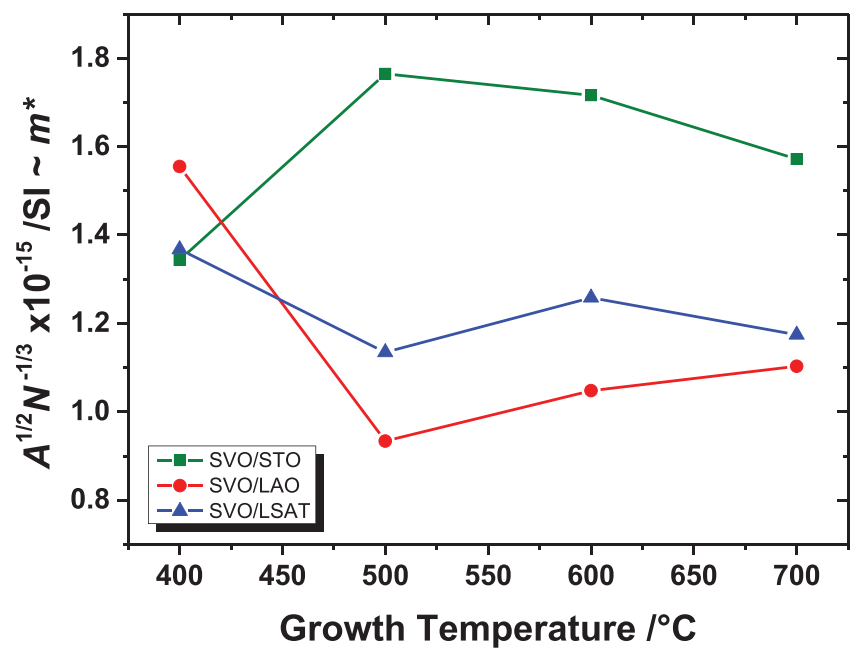

Figure 5. $A^{1 / 2} N^{-1 / 3}$ proportional to $m^{*}$ as a function of growth temperatures $T_{\mathrm{G}}$ for different substrates.

LSAT. Unfortunately, the acquisition of transmission spectra of SVO//LAO was not possible due to the presence of twined domains in the LAO substrates and the difficulty to use a reliable reference between the pristine substrate and the deposited one to realize the background in the dual beam acquisition mode.

The analysis of the transmittance shows a very high optical transparency for SVO films deposited on both STO and LSAT, even outreaching those of earlier reported data on LSAT substrates by Zhang et al. ${ }^{[1]}$ It is interesting to note here, that the films grown on STO show an important transparency at low $T_{\mathrm{G}}$ of nearly $93 \%$ going down to $80 \%$ in films grown at $700{ }^{\circ} \mathrm{C}$. This evolution may be related to the creation of active colorcenters in the STO substrate with the presence oxygen vacancies. ${ }^{[25,32]}$ In fact, it is not possible to distinguish between a loss of transparency between the STO substrate and the SVO films deposited at high temperature, so that the here reported values can only be considered as minimum values for the SVO films.

Regarding the reflectivity spectra of the SVO films shown in Figure 6b, well-known structures related to the free charge carriers (at high wavelength) and to a SVO intraband transition within the $\mathrm{V} 3 \mathrm{~d}$ conduction band located at around $3.5 \mathrm{eV}^{[21]}$ (at low wavelength) can be identified. To get more into detail, these two signatures allow to determine the screened plasma frequencies $\omega_{\mathrm{p}}$ of the films by the observed minimum. ${ }^{[33]}$ For some of the samples, $\omega_{\mathrm{p}}$ was also determined by the fitting of ellipsometry data, showing the same values and confirming therefore that the minimum of the reflectivity spectra is a good measure of $\omega_{\mathrm{p}}$. The obtained values of the films on the different substrates are shown in Figure 7. It can be clearly observed that the films on STO show higher $\omega_{\mathrm{p}}$ than the films on LSAT and LAO. There is also a certain offset between these two latter substrates, although much smaller compared to STO. Taking into account the estimation of the effective mass $m *$ from the electrical measurements (Figure 5), this dependence of $\omega_{\mathrm{p}}$ on the substrate type can be therefore understood by the changes of the electrical properties of the SVO films. In principle, it would be also possible to determine $m^{*}$ from $\omega_{\mathrm{p}}$ in TCOs, ${ }^{[34,35]}$ but the relative permittivity has to be taken into account, which may 

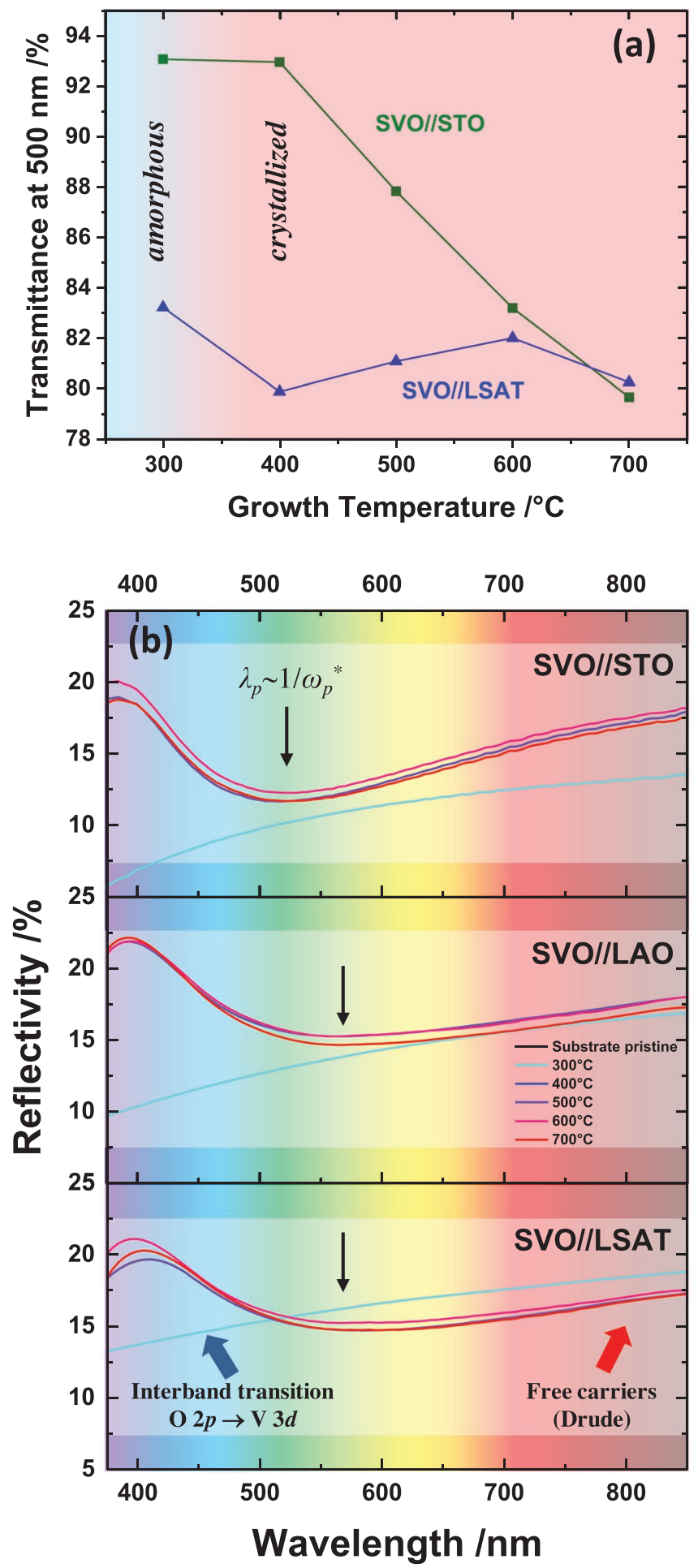

Figure 6. a) Transmittance at $500 \mathrm{~nm}$ as a function of the substrate temperature and b) reflection spectra of SVO films in the vis-NIR range for the different substrate temperatures. The black line corresponds to the pristine substrates.

depend also on the details of the growth process. Therefore, we refrain here from this calculation. However, the variation reaching around $10 \%$ of $\omega_{\mathrm{p}}$ on different substrates is reasonable regarding the variation of around a factor of two for the

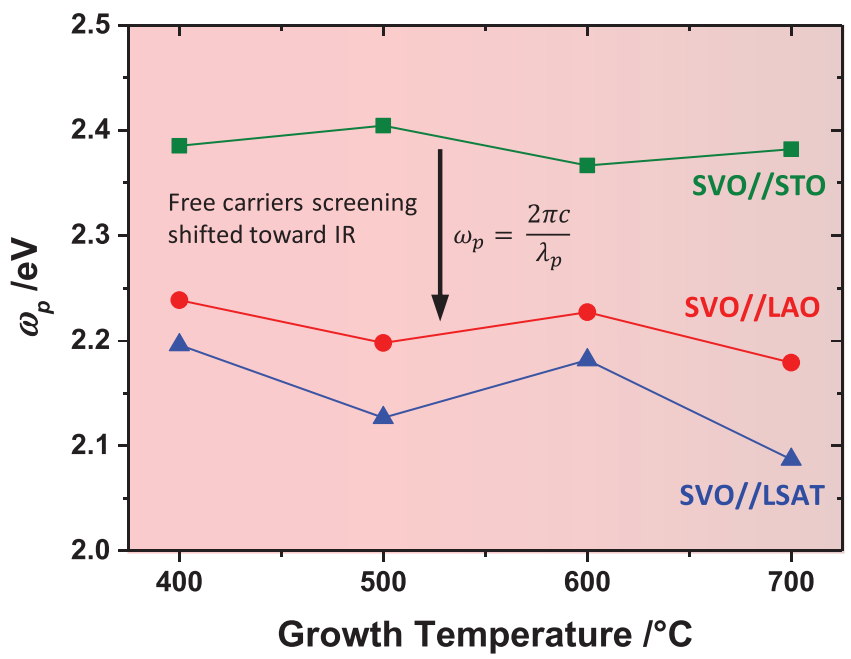

Figure 7. Plasma frequency extracted from the reflectivity spectra as a function of the growth temperature for different substrates.

estimated variation of $m *$ based on the electronic properties: $A$ is proportional to $m^{* 2}$, while $\omega_{\mathrm{p}}$ is proportional to $m^{*-1 / 2}$.

\section{Soft-Annealing Experiments on STO}

In the goal to highlight the effect of the oxygen vacancies on the properties, additional SVO films grown on STO under the same deposition conditions have been annealed under air during $20 \mathrm{~h}$ at $100{ }^{\circ} \mathrm{C}$. The annealing temperature was chosen carefully to avoid the massive oxidation of $\mathrm{SVO}$ to $\mathrm{Sr}_{3} \mathrm{~V}_{2} \mathrm{O}_{8}$ occurring above $300{ }^{\circ} \mathrm{C} \cdot{ }^{[16]}$ Figure 8 a shows the evolution of the out-of-plane lattice parameter determined by high-resolution XRD measurements of SVO before and after annealing.

After soft annealing under ambient atmosphere, the out-ofplane lattice parameter of SVO//STO decreases to approach the theoretical value of bulk SVO expected at $3.84 \AA$ (represented by dashed line in Figure $8 \mathrm{a}$ ). The swelling effect driven by the vanadium reduction in the presence of oxygen vacancies is compensated by the partial reoxidation of depleted sites under quite low temperature. The absolute variation of $c_{\mathrm{f}}$ reported in Figure $8 \mathrm{~b}$ shows that the out-of-plane deformation of the lattice is more important for films deposited at high $T_{\mathrm{G}}$. Hence, the depletion in oxygen in SVO films grown on STO under vacuum is increased by the deposition temperature.

Electrical characterizations of films summarized in Table 1 for the two uttermost $T_{\mathrm{G}}$ show a significant improvement of the transport parameters after thermal treatment. Indeed, the resistivity decreases with a variation of 0.09 and $0.55 \times 10^{-4} \Omega \mathrm{cm}$ at 400 and $700{ }^{\circ} \mathrm{C}$ while the residual resistivity is diminished of 0.05 and $0.55 \times 10^{-4} \Omega \mathrm{cm}$. At the same time, the residual resistivity ratio is nearly stable for $400{ }^{\circ} \mathrm{C}$ sample and increases notably from 1.22 up to 1.30 at $700{ }^{\circ} \mathrm{C}$. In other words, the SVO sample grown at $400{ }^{\circ} \mathrm{C}$ is less sensitive to the thermal treatment than the one deposited at $700{ }^{\circ} \mathrm{C}$.

This discrepancy can be related to an easier filling of oxygen vacancies during the treatment and consequently, a higher level in the oxygen depletion in SVO deposited at high temperature. 

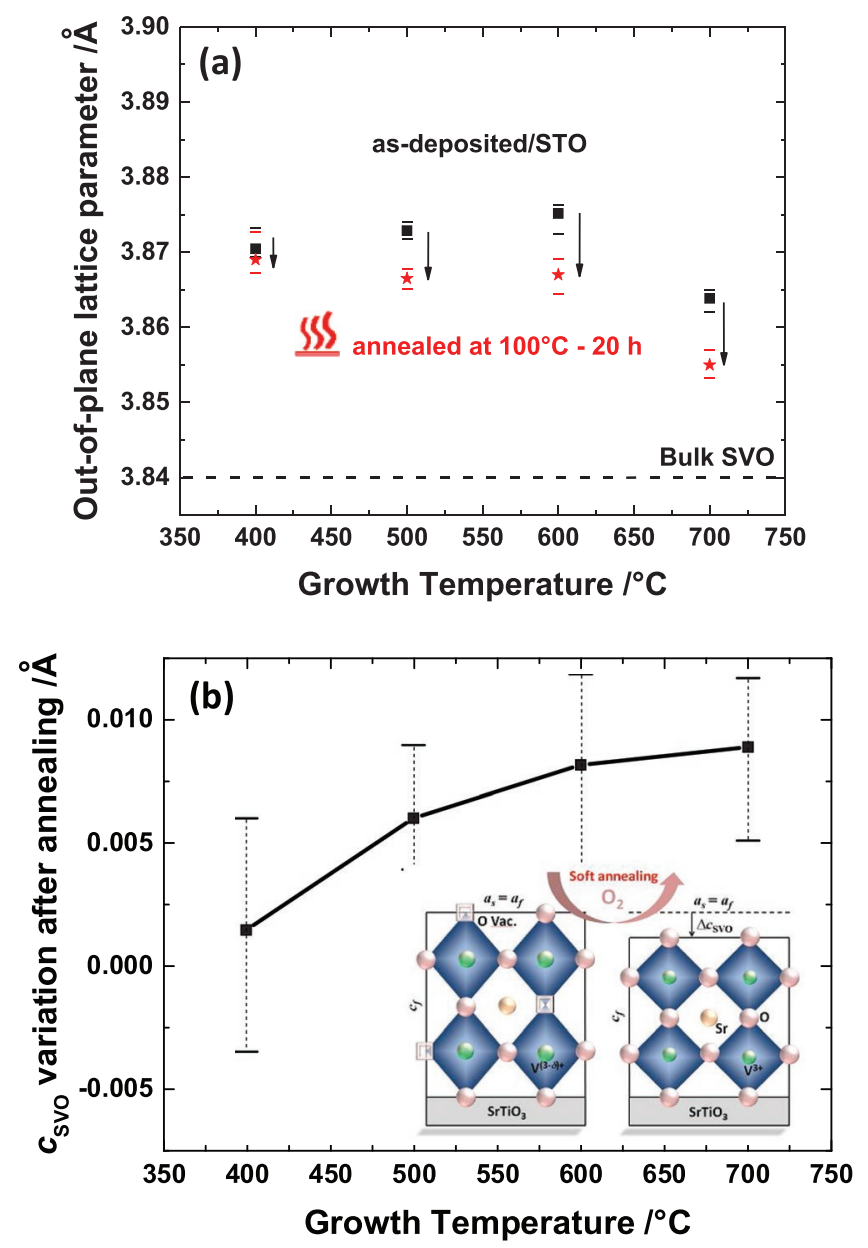

Figure 8. a) c-axis lattice parameter of SVO films grown on STO before (black squares) and after annealing (red stars). b) Absolute variation of $c$-axis after annealing.

This result is coherent with the evolution observed previously in the structural and electrical properties of SVO//STO and more precisely, the diminution of RRR indicating the oxygen loss with the growth temperature. We can notice that the carrier density and the mobility increase slightly after annealing. This variation is also more important at $700{ }^{\circ} \mathrm{C}$ compared to $400{ }^{\circ} \mathrm{C}$. Although the mobility remains less than values expected around $5-10 \mathrm{~cm}^{2} \mathrm{~V}^{-1} \mathrm{~s}^{-1}$ for bulk SVO or films deposited by $\mathrm{MBE},{ }^{[1]}$ its increase reveals the reinforcement of the conduction pathway required for the electron delocalization in the structure via the O2p-V3d exchange.

The variation of the $A$ coefficient before and after annealing is reported in Figure 9a for these samples. The fit parameter
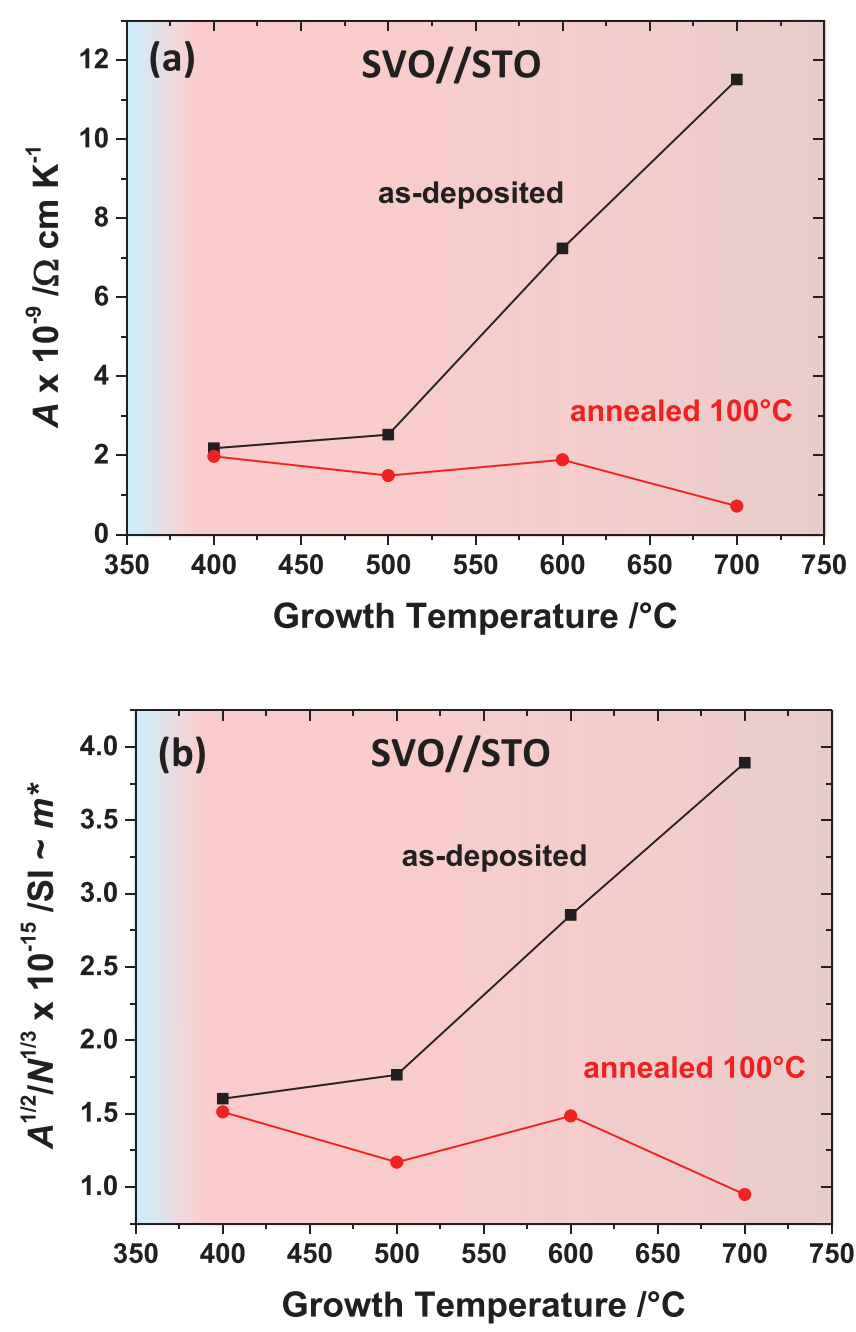

Figure 9. Variation of a) the $A$ coefficient and b) $A^{1 / 2} N^{-1 / 3}$ proportional to the electronic effective mass as a function of growth temperature before and after annealing on STO.

$A$ increases with the growth temperature, in good agreement as was previously observed for the first series of SVO//STO samples (Figure $4 \mathrm{~b}) . \mathrm{A}$ is dependent on two contributions: the average scattering probability and the electronic effective mass. ${ }^{[36]}$ As a consequence, the increase of $A$ with $T_{\mathrm{G}}$ confirms the higher structural disorder in SVO//STO films deposited at high temperature. At the same time, the effective mass increases with $T_{\mathrm{G}}$ and can be related to reinforced electronic correlations at high temperature. After soft thermal treatment, we observe a spectacular depletion of $A$ with $T_{\mathrm{G}}$ whereas it remains quasi-constant with no significant variations at $400{ }^{\circ} \mathrm{C}$.

Table 1. Transport properties of SVO//STO grown at 400 and $700{ }^{\circ} \mathrm{C}$ before and after annealing at $100{ }^{\circ} \mathrm{C}$ during 1 day.

\begin{tabular}{lccccc}
\hline SVO//STO & $\rho_{300 K}\left[\times 10^{-4} \Omega^{-1} \mathrm{~cm}^{-1}\right]$ & $\rho_{0}\left[\times 10^{-4} \Omega^{-1} \mathrm{~cm}^{-1}\right]$ & RRR & $N\left[\times 10^{22} \mathrm{~cm}^{3}\right]$ & $\mathrm{Mobility}^{2}\left[\mathrm{~cm}^{2}\left(\mathrm{~V}^{-1} \mathrm{~s}^{-1}\right)\right]$ \\
\hline $400^{\circ} \mathrm{C}$ As-deposited & 1.87 & 1.19 & 1.57 & 2.48 & 1.35 \\
$400^{\circ} \mathrm{C}$ Annealed & 1.78 & 1.14 & 1.56 & 2.55 & 1.37 \\
$700^{\circ} \mathrm{C}$ As-deposited & 2.62 & 2.14 & 1.22 & 2.09 & 1.14 \\
$700^{\circ} \mathrm{C}$ Annealed & 2.07 & 1.59 & 1.30 & 2.26 & 1.33 \\
\hline
\end{tabular}




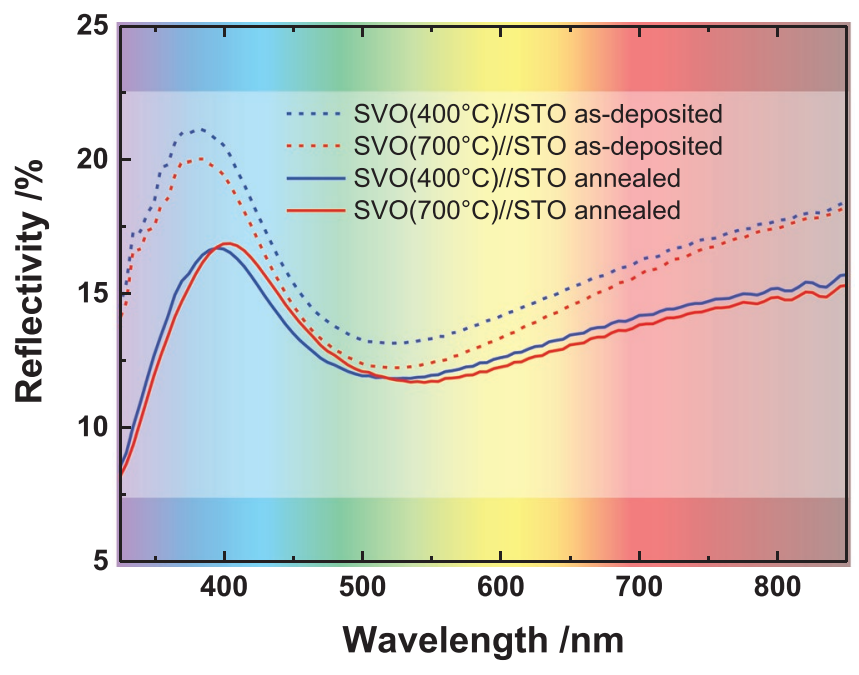

Figure 10. Reflectivity spectra of SVO films grown on STO at 400 and $700^{\circ} \mathrm{C}$ before (dashed line) and after (solid line) soft annealing at $100^{\circ} \mathrm{C}-20 \mathrm{~h}$ under atmosphere.

The effect of the thermal treatment on $m *$ is investigated by the plot of $A^{1 / 2} N^{-1 / 3}$ reported in Figure 9b. At $400{ }^{\circ} \mathrm{C}$, the estimation of $m^{*}$ is stable but is divided by a factor of four at $700{ }^{\circ} \mathrm{C}$ being comparable to those on LAO and LSAT estimated around $1.1 \times 10^{-15}$ SI (see Figure 5).

Figure 10 reports the reflectivity spectra of SVO films grown on STO at 400 and $600{ }^{\circ} \mathrm{C}$ before and after soft annealing at $100{ }^{\circ} \mathrm{C}$ during $20 \mathrm{~h}$. First, a depletion is observed in the reflectivity level of about $5 \%$ in the entire range of the probed wavelengths, i.e., including both the interband transitions and the Drude contribution. This effect, constant through the entire visible spectrum, can be related to a modification of the extreme surface of films with the possible creation of an overoxidized layer or nanostructures such as $\mathrm{Sr}_{2} \mathrm{~V}_{2} \mathrm{O}_{7}$ evidenced elsewhere by Berini et al. when $\mathrm{SrVO}_{3}$ is annealed in oxidizing conditions. ${ }^{[12]}$ Second, the annealing leads to a displacement of $\lambda_{\mathrm{p}}$ $\left(\omega_{\mathrm{p}}{ }^{*}\right)$ from 522 to $527 \mathrm{~nm}(1.19$ to $1.18 \mathrm{eV})$ for sample grown at $400{ }^{\circ} \mathrm{C}$ and from 525 to $541 \mathrm{~nm}(1.18$ to $1.15 \mathrm{eV})$ for the $700{ }^{\circ} \mathrm{C}$ one. This tendency is not consistent with the tendency of $A^{1 / 2} N^{-1 / 3}$ reported in Figure 9b, showing a decrease of $m *$ after annealing. However, it can be observed that the position of the interband transitions situated between 350 and $400 \mathrm{~nm}$ moves to high values, having a direct influence on $\omega_{\mathrm{p}}{ }^{*}$. The increase of $\omega_{\mathrm{p}}^{*}$ after annealing is therefore rather related to the effects on the lattice probed by the interband transitions, rather than the properties of the charge carriers above. Thus, conclusions about $m^{*}$ on the optical measurements are not evident in this case.

\section{Conclusion}

In conclusion, $\mathrm{SrVO}_{3}$ thin films have been grown by PLD on STO, LSAT, and LAO perovskite substrates and their structural, electrical and optical properties have been examined. First, $\mathrm{SrVO}_{3}$ crystallizes surprisingly from $400{ }^{\circ} \mathrm{C}$ on whatever the used substrate with however a remarkably high structural quality in the case of in-plane tensile strain while a compressive strain limits the local order in the crystalline structure.

Second, the resistivity of films remains around $(\approx 1-2) \times 10^{-4} \Omega \mathrm{cm}$, indicating that transport properties are not too dependent on the substrate and the growth temperature, even if slight variations are observed. In particular, we have shown the depletion of oxygen vacancies in SVO deposited onto STO substrate at high temperature by postannealing experiments. The sub-stoichiometry in oxygen can be understood by an interdiffusion effect at the SVO/STO interface during the deposition under reducing conditions where STO is unstable.

Globally, the properties of SVO are not affected strongly by strains induced by the substrate, oxygen depletion in films seems to play a more important role on the conduction. However, the changes in the electronic properties indicate a higher effective electronic mass $m^{*}$ for the films grown in STO, resulting also in a higher screened plasma frequency $\omega^{*}$, compared to the other substrates.

In the objective to design and control tailored optical properties ensuring a good transparency through the visible range, an adapted system based on SVO film has to show a low plasma frequency, out of the visible range. As the plasma frequency is proportional to $\left(N / m^{*}\right)^{1 / 2}$, this condition can be accomplished by increasing $m^{*}$ by introducing, for example, oxygen vacancies in the structure via a chemical exchange with the substrate or by the tensile strains. However, important oxygen depletion affects the electronic transport of films and a compromise between the conduction and the transparency should be found.

\section{Experimental Section}

SVO thin films have been grown on double side polished (001) oriented STO, LSAT, and LAO substrates by PLD. Prior to deposition, substrates $(5 \times 5 \times 0.5 \mathrm{~mm})$ were successively cleaned in acetone and ethanol ultrasonic baths. A KrF excimer laser $\left(\lambda_{\text {laser }}=248 \mathrm{~nm}\right)$ was used with a repetition rate of $3 \mathrm{~Hz}$ and a laser fluence of $\approx 1.6 \mathrm{~J} \mathrm{~cm} \mathrm{~cm}^{-2}$ focusing on a $\mathrm{Sr}_{2} \mathrm{~V}_{2} \mathrm{O}_{7}$ polycrystalline target prepared by standard solid-state reaction. The deposition was carried out under vacuum to induce the reduction of the target material and to avoid overoxidation of the SVO films, known to lead to the presence of higher oxides as $\mathrm{Sr}_{3} \mathrm{~V}_{2} \mathrm{O}_{8} \cdot{ }^{[12]}$ The residual deposition pressure at the growth temperature was $1 \times 10^{-6} \mathrm{mbar}$, while the base pressure is $1 \times 10^{-7} \mathrm{mbar}$. The target-substrate distance was set at $50 \mathrm{~mm}$ in order to achieve a deposition rate of $0.09 \AA$ per pulse and the $\mathrm{SrVO}_{3}$ thickness was fixed to be around $40 \mathrm{~nm}$ for all samples by adjusting the deposition time. In order to investigate the influence of the temperature on the crystallization of $\mathrm{SrVO}_{3}$ films, the substrate temperature $\left(T_{\mathrm{G}}\right)$ has been varied from 300 to $700^{\circ} \mathrm{C}$.

The structural properties and the thickness of the samples were characterized by XRD with a Bruker D8 Discover diffractometer operating with monochromatic $\mathrm{Cu} \mathrm{K} \mathrm{K}_{\alpha 1}$ radiation $(\lambda=1.5406 \AA)$. The temperature dependence of transport properties was determined by the four probe method from 5 to $300 \mathrm{~K}$ using the Van der Pauw configuration in a physical properties measurement system by Quantum Design. To determine the carrier concentration and the mobility of carriers, Hall measurements were performed in the same temperature range by applying perpendicularly to the film plane a magnetic field within a range from -9 to $+9 \mathrm{~T}$. The optical properties were measured in the UV-vis-near infrared range using a Lambda 1050 PerkinElmer spectrophotometer operating in reflection mode by means of the universal reflectance accessory and transmission mode. For all substrates, a pristine substrate was taken as reference. 
In order to ensure the absence of a possible deviation in the cationic contents of SVO films, chemical analyses have been performed by RBS with $1.7 \mathrm{MeV} \mathrm{He}^{+}$ions (not shown here). The quantification of the $\mathrm{Sr} / \mathrm{V}$ ratio indicates a maximum variation from the stoichiometric value $\mathrm{Sr} / \mathrm{V}=1 \%$ of $6 \%$ between 400 and $700{ }^{\circ} \mathrm{C}$ without a clear dependence on the growth temperature or the used substrate. It thus confirms an excellent transfer of the cation stoichiometry of the target under the deposition conditions used. This discards a possible effect of the cationic composition on the structural or functional properties of the films.

\section{Acknowledgements}

The authors thank the Labex EMC3 (Energy Materials and Clean Combustion Center) for its financial support in the framework of the COTRA project. The authors thank also M. Boisserie and C. Frilay for important technical help, on the electrical and optical measurements, respectively.

\section{Conflict of Interest}

The authors declare no conflict of interest.

\section{Keywords}

correlated materials, epitaxy, thin films, transparent conducting oxides, vanadates

[1] L. Zhang, Y. Zhou, L. Guo, W. Zhao, A. Barnes, H.-T. Zhang, C. Eaton, Y. Zheng, M. Brahlek, H. F. Haneef, N. J. Podraza, M. H. W. Chan, V. Gopalan, K. M. Rabe, R. Engel-Herbert, Nat. Mater. 2016, 15, 204.

[2] A. Boileau, A. Cheikh, A. Fouchet, A. David, R. Escobar-Galindo, C. Labbé, P. Marie, F. Gourbilleau, U. Lüders, Appl. Phys. Lett. 2018, 112, 021905.

[3] M. K. Stewart, J. Liu, R. K. Smith, B. C. Chapler, C.-H. Yee, R. E. Baumbach, M. B. Maple, K. Haule, J. Chakhalian, D. N. Basov, J. Appl. Phys. 2011, 110, 033514.

[4] S. Autier-Laurent, B. Mercey, D. Chippaux, P. Limelette, C. Simon, Phys. Rev. B 2006, 74, 195109.

[5] Q. Gan, R. A. Rao, C. B. Eom, J. L. Garrett, M. Lee, Appl. Phys. Lett. 1998, 72, 978.

[6] G. Sclauzero, K. Dymkowski, C. Ederer, Phys. Rev. B 2016, 94, 245109.

[7] Z. Zhong, M. Wallerberger, J. M. Tomczak, C. Taranto, N. Parragh, A. Toschi, G. Sangiovanni, K. Held, Phys. Rev. Lett. 2015, 114, 246401.
[8] K. Yoshimatsu, T. Okabe, H. Kumigashira, S. Okamoto, S. Aizaki, A. Fujimori, M. Oshima, Phys. Rev. Lett. 2010, 104, 147601.

[9] J. A. Moyer, C. Eaton, R. Engel-Herbert, Adv. Mater. 2013, 25, 3578.

[10] M. Gu, S. A. Wolf, J. Lu, J. Phys. D: Appl. Phys. 2018, 51, 10 LT01.

[11] A. Fouchet, M. Allain, B. Bérini, E. Popova, P.-E. Janolin, N. Guiblin, E. Chikoidze, J. Scola, D. Hrabovsky, Y. Dumont, N. Keller, Mater. Sci. Eng. B 2016, $212,7$.

[12] B. Bérini, V. Demange, M. Bouttemy, E. Popova, N. Keller, Y. Dumont, A. Fouchet, Adv. Mater. Interfaces 2016, 3, 1600274.

[13] D.-W. Kim, D. H. Kim, T. W. Noh, K. Char, J.-H. Park, K.-B. Lee, H.-D. Kim, J. Appl. Phys. 2000, 88, 7056

[14] P. Dougier, J. C. C. Fan, J. B. Goodenough, J. Solid State Chem. 1975, 14, 247.

[15] M. Onoda, H. Ohta, H. Nagasawa, Solid State Commun. 1991, 79 281.

[16] J. Macías, A. A. Yaremchenko, J. R. Frade, J. Alloys Compd. 2014, 601, 186

[17] J. R. Petrie, C. Mitra, H. Jeen, W. S. Choi, T. L. Meyer, F. A. Reboredo, J. W. Freeland, G. Eres, H. N. Lee, Adv. Funct. Mater. 2016, 26, 1564.

[18] T. Mayeshiba, D. Morgan, Phys. Chem. Chem. Phys. 2015, 17, 2715.

[19] M. J. Rey, P. Dehaudt, J. C. Joubert, B. Lambert-Andron, M. Cyrot, F. Cyrot-Lackmann, J. Solid State Chem. 1990, 86, 101.

[20] K.-J. Range, F. Rau, U. Klement, Z. Naturforsch., B 1991, 46, 1315.

[21] H. Makino, I. H. Inoue, M. J. Rozenberg, I. Hase, Y. Aiura, S. Onari, Phys. Rev. B 1998, 58, 4384.

[22] T. Maekawa, K. Kurosaki, S. Yamanaka, J. Alloys Compd. 2006, 426, 46.

[23] D. R. Lide, CRC Handbook of Chemistry and Physics: A Ready-Reference Book of Chemical and Physical Data: 2006-2007, CRC Press, Boca Raton, FL 2006.

[24] A. Frye, R. H. French, D. A. Bonnell, Z. Metallkd. 2003, 94, 226.

[25] H. N. Lee, S. S. Ambrose Seo, W. S. Choi, C. M. Rouleau, Sci. Rep. 2016, 6, 19941.

[26] A. B. Posadas, K. J. Kormondy, W. Guo, P. Ponath, J. Geler-Kremer, T. Hadamek, A. A. Demkov, J. Appl. Phys. 2017, 121, 105302.

[27] G. Herranz, M. Basletić, O. Copie, M. Bibes, A. N. Khodan, C. Carrétéro, E. Tafra, E. Jacquet, K. Bouzehouane, A. Hamzić, A. Barthélémy, Appl. Phys. Lett. 2009, 94, 012113.

[28] D. L. Ritums, N. J. Wu, X. Chen, D. Liu, A. Ignatiev, AIP Conf. Proc. 1998, 420, 672.

[29] P. W. Yip, K. H. Wong, MRS Online Proc. Libr. Arch. 2000, 623, 347.

[30] V. Giannakopoulou, P. Odier, J. M. Bassat, J. P. Loup, Solid State Commun. 1995, 93, 579

[31] I. H. Inoue, O. Goto, H. Makino, N. E. Hussey, M. Ishikawa, Phys. Rev. B 1998, 58, 4372.

[32] J. Hanzig, B. Abendroth, F. Hanzig, H. Stöcker, R. Strohmeyer, D. C. Meyer, S. Lindner, M. Grobosch, M. Knupfer, C. Himcinschi, U. Mühle, F. Munnik, J. Appl. Phys. 2011, 110, 064107.

[33] D. N. Basov, R. D. Averitt, D. van der Marel, M. Dressel, K. Haule, Rev. Mod. Phys. 2011, 83, 471.

[34] D. Pines, D. Bohm, Phys. Rev. 1952, 85, 338.

[35] S. Franzen, J. Phys. Chem. C 2008, 112, 6027.

[36] M. Núñez-Regueiro, G. Garbarino, M. D. Núñez-Regueiro, J. Phys.: Conf. Ser. 2012, 400, 022085. 